\title{
A CASE REPORT ON SARCOIDOSIS
}

\author{
DEB SR ${ }^{1}$, SARKAR R $^{2}$, HASAN P ${ }^{3}$, MUKERRAMA SM ${ }^{4}$, BARAL P ${ }^{5}$, KABIR A $^{6}$, HOSSAIN M$^{7}$

\begin{abstract}
:
Sarcoidosis is a multisystem disease that may involve almost any organ system. Infiltration with non-caseating granulomas is the hallmark of the disease, and it may result in various clinical manifestations. Since the first description of the disease by British dermatologist Jonathan Hutchison in 1869, sarcoidosis has been seen in almost every part of the world and continues to engender considerable interest among clinicians because of its unknown aetiology, protean manifestations, and variable course and prognosis. Here we report a 65-year old woman who presented to us with a rather atypical presentation of sarcoidosis- subcutaneous nodules. Later, on subsequent diagnostic work-up, she was found to have bilateral hilar and mediastinal lymphadenopathy and pulmonary reticular lesions. It is a bit unusual to have only subcutaneous nodules as the presenting complaint of sarcoidosis without any prominent systemic and respiratory symptoms, which is what prompted this report.
\end{abstract}

Key words: Sarcoidosis, subcutaneous nodules.

J Dhaka Med Coll. 2013; 22(2) : 225-228.

\section{Introduction:}

Sarcoidosis is an inflammatory disease characterized by the presence of noncaseating granulomas. While the disease can affect virtually every organ of the body, the lung is most commonly affected. Other organs commonly affected are the liver, skin and eye. Cutaneous involvement occurs in 20 to 35 percent of patients with systemic sarcoidosis and may occur without systemic involvement ${ }^{1}$. This involvement may occur at any stage of the disease though it is most often present at the onset and may even be the presenting complaint ${ }^{2}$. Despite multiple investigations, the cause of sarcoidosis remains unknown. Currently, the most likely aetiology is an infectious or noninfectious environmental agent that triggers an inflammatory response in a genetically susceptible host. Sarcoidosis is seen worldwide, with the highest prevalence reported in the Nordic population ${ }^{3}$.
Sarcoidosis often occurs in young, otherwise healthy adults. However, it has become clear that a second peak in incidence develops around age $60^{3}$. Women appear to be slightly more susceptible than men. Although most cases of sarcoidosis are sporadic, a familial form of the disease exists. At least $5 \%$ of patients with sarcoidosis have a family member with sarcoidosis ${ }^{3}$.

\section{Case Report:}

A 65-year old hypertensive, diabetic housewife, hailing from Comilla district, presented with bilateral leg swelling for 2 months and multiple nodular swellings on forearms, wrists and hands for about same duration. The patient states that she was reasonably well 2 months back. Then she developed swelling in her both legs, which was not associated with any pain, redness or local rise of temperature. Then, after about 7 days, she noticed some nodular swellings on her forearms, wrists and hands (Fig. 1).

1. Dr. Sudip Ranjan Deb, Assistant Professor, Department of Medicine, Dhaka Medical College, Dhaka.

2. Dr. Ratul Sarkar, Medical Officer, Department of Medicine, Dhaka Medical College Hospital, Dhaka.

3. Dr. Pratyay Hasan, Medical Officer, Department of Medicine, Dhaka Medical College Hospital, Dhaka.

4. Dr. Shegufta Mishket Mukerrama, Department of Medicine, Dhaka Medical College Hospital, Dhaka.

5. Dr. Prianka Baral, Medical Officer, Department of Medicine, Dhaka Medical College Hospital, Dhaka.

6. Dr. Ahmedul Kabir, Associate Professor, Department of Medicine, Dhaka Medical College Hospital, Dhaka.

7. Dr. Mahbub Hossain, Medical Officer, Department of Medicine, Dhaka Medical College Hospital, Dhaka.

Correspondence: Dr. Sudip Ranjan Deb, Assistant Professor, Department of Medicine, Dhaka Medical College, Dhaka. Cell Phone: +8801714963196, Email: sudipranjandev@yahoo.com 


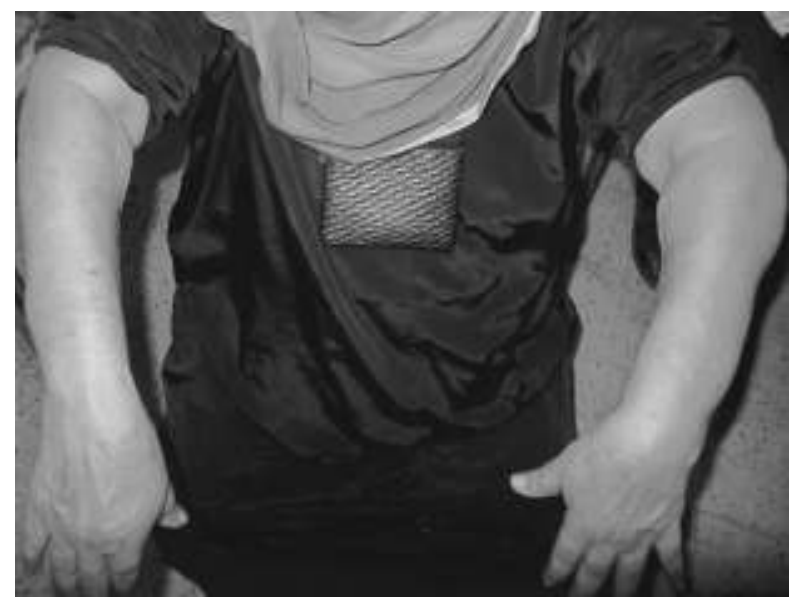

Fig.-1: Photograph showing subcutaneous nodules.

These swellings are neither painful nor associated with any local redness or rise of temperature. She complains of occasional respiratory distress, especially on exertion. The distress does not aggravate on lying position. She gives no history of fever, cough, chest pain, or swelling in any other part of the body, except the parts aforementioned. She tells us that she has lost her appetite over the last 2 months. She also feels unusually fatigued now-a-days. But there is no history of recent significant weight loss, nausea, vomiting, or abdominal pain. Her bowel and bladder habits are normal. She gives no history of headache, seizure, loss of consciousness, tingling or burning sensation in the extremities, or any visual disturbance. She previously took amlodipine as antihypertensive for several years. She gives no history of contact with any tuberculosis patient and she has no family member affected by the type of illness she is suffering from. Physical examination revealed multiple subcutaneous nodules on forearms, wrists and hands. The nodules were firm, nontender, with smooth surface and indistinct margin. The largest one measured about $4 \times 3$ $\mathrm{cm}$ in size. Some of the nodules were attached to the overlying skin and underlying structure, while others were not. There was no erythema, ulceration or hypo- or hyperpigmentation of the skin over the nodules. The patient did not report any injuries on the sites of lesions. Her blood pressure was $130 / 70 \mathrm{~mm}$ of $\mathrm{Hg}$ (on treatment with antihypertensive medication), and there were bilateral pitting pedal oedema, and coarsecrackles in left $7^{\text {th }}$ to $9^{\text {th }}$ intercostal spaces. Apart from these, there are no abnormal findings on physical examination. Her complete blood count, urine R/E, serum creatinine, and ECG showed no abnormality. The diagnosis of cutaneous sarcoidosis was confirmed by histological examination of skin biopsy specimens. The skin samples were obtained from the nodules on the left forearm. Sections made from both of the samples revealed (Fig. 2) many well-formed non-caseating granulomas made of epithelioid cells and giant cells. Lymphocytes were small in number. Afterwards, a chest x-ray and CT scan of chest was done. Chest X-ray showed right hilar lymphadenopathy, and the CT scan of chest revealed enlarged discrete lymph nodes in the mediastinum (peritracheal, carinal, adjacent to arch of the aorta and posterior mediastinum) and in both hilar regions. Fine reticular densities were seen in both lungs predominantly in the upper lobes. The tuberculin skin test was negative. All these findings supported the diagnosis of sarcoidosis. She is currently on treatment with antihypertensive (e.g. losartan potassium), antidiabetic (e.g. metformin and insulin), and corticosteroids.

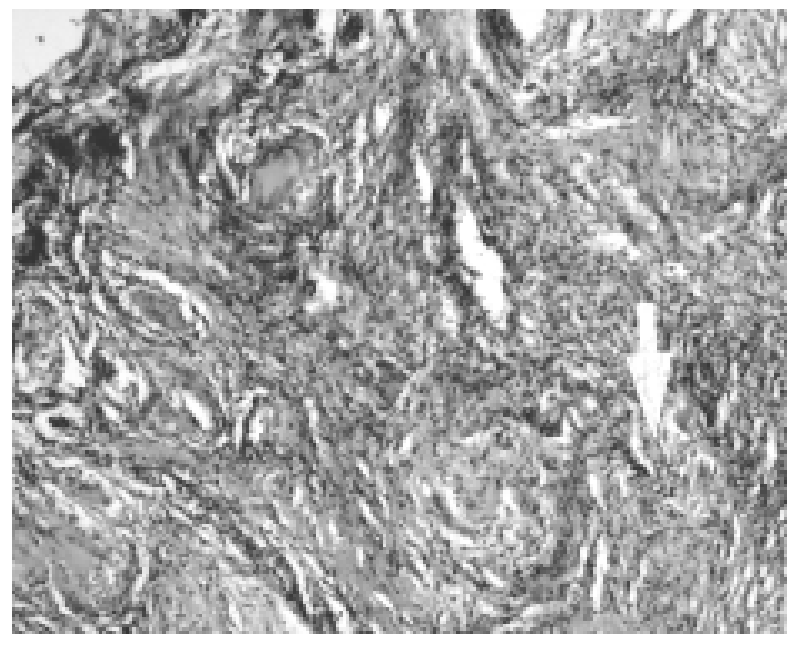

Fig. 2: Photomicrograph showing well-formed noncaseating granulomas. 


\section{Discussion:}

Sarcoidosis is a granulomatous disease of unknown aetiology that is suspected to be the product of a combination of genetic, immunologic, and environmental factors. The first reported association between sarcoidosis and specific gene products was the association between class I HLA-B8 antigens and acute sarcoidosis ${ }^{4}$. Subsequently, HLA class II antigens, encoded by .HLA-DRB1 and DQB1 alleles, have been consistently associated with sarcoidosis ${ }^{5,6}$. Environmental exposuresto insecticides and mold have been associated with an increased risk for disease ${ }^{3}$. A cardinal feature of sarcoidosis is the presence of $\mathrm{CD}^{+}$ $\mathrm{T}$ cells that interact with antigen presenting cells to initiate the formation and maintenance of granulomas ${ }^{7}$. Sarcoidosis also presents as an 'immune paradox': despite extensive local inflammation, anergy may develop, as indicated by the immune response to tuberculin. Expansion of CD25 bright regulatory T cells, a subgroup of $\mathrm{CD}^{+} \mathrm{T}$ lymphocytes in active sarcoidosis may account for this anergy by abolishing interleukin-2 production and strongly inhibiting $\mathrm{T}$ cell proliferation ${ }^{8}$. The presentation of sarcoidosis ranges from patients who are asymptomatic to those with organ failure. In countries where routine chest $\mathrm{x}$-ray screening is performed, $20-30 \%$ of pulmonary cases are detected in asymptomatic individuals ${ }^{8}$. Respiratory complaints including cough and dyspnoea are the most common presenting symptoms. In fact, lung involvement occurs in more than $90 \%$ of sarcoidosis patients. Symptoms related to cutaneous and ocular disease are the next two common complaints. Skin lesions are often nonspecific. The frequency of various skin lesions in sarcoidosis may be present: erythema nodosum $(20.5 \%)$, cutaneous plaques $(4.3 \%)$, subcutaneous nodules (4.3\%), maculopapular rash $(3.7 \%)$, scar lesions $(2.9 \%)$, lupus pernio $(2.7 \%)$, and psoriaform lesions $(0.9 \%)^{9}$. Since the skin lesions are readily observed, the patient and treating physician are often led to a diagnosis. This is what exactly happened in our case. The patient's subcutaneous nodules led us to take biopsy from those lesions, which revealed non-caseating granulomas suggestive of sarcoidosis. Then we retrospectively proceeded to find any other system involvement with $\mathrm{X}$-ray and CT scan of the chest, which showed lung involvement, manifested by bilateral hilar and mediastinal lymphadenopathy and diffuse parenchymal reticular lesions. Sarcoidosis may also involve the heart, liver, spleen, kidneys and the nervous system, among other organs. There is no single diagnostic test for sarcoidosis. The diagnosis of sarcoidosis is based on the following criteria: a) a compatible clinical and/ or radiographic picture, b) histological evidence of non-caseating granulomas and c) exclusion of other conditions with similar histology ${ }^{10}$. The most common abnormalities on plain chest radiographs are bilateral, symmetric hilar adenopathy with or without lung infiltrates. CT scans, especially using high resolution technique provide much greater detail on chest radiographs Common patterns in sarcoidosis include widespread pulmonary nodules, infiltrates with a bronchovascular and sub-pleural distribution, thickened interlobular septa etc. Intrathoracic adenopathy is also more often detected on CT. Gallium-67 scanning may be of value in the initial diagnostic evaluation, but the additional cost and radiation exposure with gallium scanning are not warranted in most cases ${ }^{11}$. Most recent data indicate that serum angiotensin converting enzyme (ACE) levels are neither sufficiently sensitive nor specific to confirm a diagnosis of sarcoidosis although they may have some value as supportive evidence for or against the diagnosis ${ }^{12}$. However, most patients with sarcoidosis require histologic confirmation for diagnosis. Since sarcoidosis is a multisystem disorder, evidence of granulomatous inflammation in at least 2 organs is required to distinguish it from granulomatous disorders of individual organs ${ }^{12,13}$. However, biopsy confirmation from one organ is deemed sufficient if compatible clinical, laboratory, or radiologic findings are consistent with the diagnosis in at least one additional organ and alternative diagnoses have been excluded ${ }^{12,13}$. The choice of biopsy site should be guided by what is least invasive and most likely to yield diagnostic material. Enlarged peripheral lymph nodes, skin involvement, and conjunctival nodules permit minimally invasive procedures. Bronchoscopy is often the diagnostic procedure of choice in patients with intrathoracic involvement. Besides, lung parenchyma can be sampled by trans-bronchial lung biopsy. Mediastinal and hilar lymph nodes may be accessed with trans-bronchial needle 
aspiration and endobronchialultrasound, though mediastinoscopy still remains the gold standard. Analysis of bronchoalveolar lavage fluid can be helpful to exclude conditions which figure strongly in the differential diagnosis, such as tuberculosis and fungal infections. Because of the invasiveness of the procedures listed above, and because we found involvement of two most common organs affected by sarcoidosis, (skin involvement proved by biopsy, and the lung by x-ray and CT scan of chest) we did not do any of these invasive procedures. In difficult cases, FDG-PET scan and gadolinium enhanced scan may help to identify occult sites (e.g. heart, nervous system) involved by sarcoidosis Systemic corticosteroids are the drugs of choice for the treatment of sarcoidosis, although no clear consensus exists regarding when to start treatment, the correct doses and or how long treatment should be maintained ${ }^{14}$. Other alternative treatments such as immunosuppressive agents (e.g. methotrexate, azathioprine, cyclophosphamide, leflunomide), cytokine inhibitors (e.g. thalidomide, pentoxifylline), antimalarial agents such as chloroquine are only indicated when the sarcoidosis does not respond to conventional treatment with oral corticosteroids, in patients with intolerance to corticosteroids, or in order to reduce he dosage of corticosteroids ${ }^{14}$.

\section{Conclusion:}

'Any complaint made by the patient should not be overlooked'-our case demonstrated how true the statement is. To make any diagnosis, an inquisitive mind is necessary. Subcutaneous nodules in a 65-year old lady, though not a common presentation of sarcoidosis, ultimately opened the door of diagnosing the case. Later, with chest $\mathrm{x}$-ray and CT scan of chest, it turned out that it was indeed a case of sarcoidosis. This diagnosis was important in two ways. First, we could give her proper treatment with reassurance that two thirds of patients with this disease have a remission within a decade after diagnosis, with few or no consequences ${ }^{15}$. Remission occurs for more than half of patients within 3 years. A minority (less than $5 \%$ ) of patients die from sarcoidosis without treatment, usually as a result of pulmonary fibrosis with respiratory failure or of cardiac or neurologic involvement ${ }^{15}$. Hopefully, she would not be among those unfortunate patients, as the treatment was started earlier. Second, this diagnosis was an eye-opener for us, showing how much atypically a disease can present.

\section{References}

1. Samtsov A. Cutaneous sarcoidosis. Int J Dermatol 1989; 31: 385-91.

2. Katta R. Cutaneous sarcoidosis: a dermatologic masquerader. Am Fam Physician 2002; 65: 1581-4.

3. Baughman R, Lower E. Sarcoidosis. In: Longo D, Kasper D, Jameson J, Fauci A, Hauser S, Loscalzo $\mathrm{J}$, editors. Harrison's principles of internal medicine. $18^{\text {th }}$ ed. New York: The McGraw-Hill; 2012: p.2806-7.

4. Brewerton D, Cockburn C, James D, James D, Neville E. HLA antigens in sarcoidosis. Clin Exp Immunol 1977; 27: 227-29.

5. Iannuzzi M, Maliarik M, Poisson L, Rybicki B. Sarcoidosis susceptibility and resistance HLADQB1 alleles in African Americans. Am J Respir Crit Care Med 2003; 167: 1225-31.

6. Rossman M, Thompson B, Frederick M. HLADRB 1*1101: a significant risk factor for sarcoidosis in blacks and whites. Am J Hum Genet 2003; 73: 720-35.

7. Agostini C, Adami F, Semenzato G. New pathogenetic insights into the sarcoid granuloma. Curr Opin Rheumatol 2000; 12: 71-6.

8. Miyara M, Amoura Z, Parizot C. The immune paradox of sarcoidosis and regulatory $\mathrm{T}$ cells. $\mathrm{J}$ Exp Med 2006; 203: 359-70.

9. Yanardag H, Pamuk O, Karayel T. Cutaneous involvement in sarcoidosis:analysis of the features in 170 patients. Respir Med. 2003; 97: p. 978-82.

10. Gerolemou L, Smith P. Basic diagnostic approaches in sarcoidosis. In: Motamedi M, ed. Sarcoidosis diagnosis and management. $1^{\text {st }} \mathrm{ed}$. Rijeka: InTech; 2011. p.37.

11. Gerolemou L, Smith P. Basic diagnostic approaches in sarcoidosis. In Motamedi M, ed. Sarcoidosis diagnosis and management. $1^{\text {st }}$ ed. Rijeka: InTech; 2011. p. 40-1.

12. Baughman R, Culver D, Judson M. A concise review of pulmonary sarcoidosis. Am Rev Respir Crit Care Med 2011; 183: 573-81.

13. Judson M. The diagnosis of sarcoidosis. Clin Chest Med 2008; 29: 415-27.

14. Jara-Palomares L, Cabellaro-Eraso CB, RodriguezPortal J. Updated guidelines for the treatment of pulmonary sarcoidosis. In: Motamedi M, ed. Sarcoidosis diagnosis and management. $1^{\text {st }}$ ed. Rijeka: InTech; 2011. p. 125-6.

15. Iannuzzi M, Rybicki B, Teirstein A. Sarcoidosis. N Engl J Med 2007; 357: 2153-65. 